\title{
UNIVERSAL SCALING OF BASIC PROPERTIES OF THE HEAVY-FERMION SUPERCONDUCTORS
}

\section{J. Karbowski AND J. SPaleK}

Institute of Theoretical Physics, Warsaw University, Hoża 69, 00-681 Warszawa, Poland

We show that the properties of the heavy-electron superconducting state induced by the interorbital kinetic exchange scale with the effective mass renormalization $m^{*} / m_{0} \sim 1 / T_{\mathrm{K}}$. Explicitly, the pairing potential $\tilde{J} \sim$ $J\left(m_{0} / m^{*}\right) \ln ^{2}\left(m_{0} / m^{*}\right)$, where $J$ is the magnitude of the bare Kondo coupling; the coherence length $\xi \sim T_{\mathrm{K}} / T_{\mathrm{c}}$, where $T_{\mathrm{c}}$ is the transition temperature, whereas the penetration depth $\lambda \sim\left(m^{*} / m_{0}\right)^{1 / 2}$ so that $\lambda / \xi \gg 1$. We also determine the scaling of magnetic critical fields.

PACS numbers: $74.70 . \mathrm{Tx}, 75.30 . \mathrm{Mb}, 71.28 .+\mathrm{d}$

In this paper we predict a scaling of fundamental parameters characterizing a heavy-fermion superconductor, which extends the earlier analysis for the normal state [1]. This goal is achieved by considering the lattice Anderson model in which first order corrections in $1 / U$, where $U$ is the magnitude of the intraatomic $f-f$ interaction, have been included [2] so as to generate an interorbital (hybrid) pairing. In this manner, both the Fermi liquid state of almost localized electrons, as well as their superconducting properties are obtained within a single framework. An earlier treatment [3] of superconductivity within the lattice Anderson model in the $U=\infty$ limit required higher-order $\left(1 / N^{2}\right)$ correction to the mean-field slave-boson picture of the heavy electrons. Here, a stable superconducting phase appears already in the mean-field approximation for the pairing part and provides a universal scaling with the mass renormalization $m^{*} / m_{0}$, as discussed below.

We start from the effective Hamiltonian derived earlier [2] to the first nontrivial order in $V / U$, which was rederived in the slave boson representation of Zou and Anderson [4] and takes the form

$$
\begin{aligned}
\tilde{\mathcal{H}}= & \sum_{m n \sigma} t_{m n} c_{m \sigma}^{+} c_{n \sigma}+\varepsilon_{f} \sum_{i \sigma} f_{i \sigma}^{+} f_{i \sigma}+\sum_{i m \sigma}\left(V_{i m} e_{i} f_{i \sigma}^{+} c_{m \sigma}+\text { H.c. }\right) \\
& -\sum_{i m}\left(2 V_{i m}^{*} V_{i n} / \bar{U}\right) b_{i m}^{+} b_{i n}+\sum_{i} \lambda_{i}\left(e_{i}^{+} e_{i}+\sum_{\sigma} f_{i \sigma}^{+} f_{i \sigma}-1\right)
\end{aligned}
$$

with

$$
b_{i m}^{+} \equiv\left(f_{i \uparrow}^{+} c_{m \downarrow}^{+}-f_{i \downarrow}^{+} c_{m \uparrow}^{+}\right) / \sqrt{2},
$$

and $\bar{U} \equiv U-\tilde{\varepsilon}_{f}+\mu$.

The first three terms comprise the Anderson lattice model in $U=\infty$ limit. This formulation involves a single scalar boson and has been studied extensively in 
the last decade [1] in the limit $d_{i}^{+} d_{i} \equiv 0$. The fourth term expresses the interorbital spin singlet pairing introduced before $[2,5]$, here reformulated in slave boson language. The last term contains a set of Lagrange multipliers $\left\{\lambda_{i}\right\}$ reflecting the local constraint which is imposed at every $f$-site due to the introduction of extra Bose field $e$.

One can easily diagonalize the single-particle part of (1) in the mean-field approximation and obtain the usual eigenenergies

$$
E_{k_{\alpha}}=(1 / 2)\left\{\varepsilon_{k}+\tilde{\varepsilon}_{f}-2 \mu+\alpha\left[\left(\varepsilon_{k}-\tilde{\varepsilon}_{f}\right)^{2}+(2 V e)^{2}\right]^{\frac{1}{2}}\right\},
$$

where $\alpha= \pm 1$. The renormalized $f$-level position $\tilde{\varepsilon}_{f}$ is very close to the Fermi level and at $T=0$ yields

$$
\tilde{\varepsilon}_{f}-\mu \sim(W / 2) \exp \left(-\frac{\mu-\varepsilon_{f}}{V^{2} \rho_{0}}\right)
$$

where the bare band spans from $-W / 2$ to $W / 2$ and is assumed as featureless. The above energy difference is defined as $k_{\mathrm{B}} T_{\mathrm{K}}$, where $T_{\mathrm{K}}$ is customarily called the effective Kondo temperature [1]. The density of quasiparticle states at $\mu$ is then $\rho(\mu)=1 / 2 k_{\mathrm{B}} T_{\mathrm{K}}$ and therefore very large.

We now present the scaling of quantities with $T_{\mathrm{K}}$ in the superconducting phase. For that purpose we consider the case for which the number of particles is $n \leq 2$ per site so that only the lower hybridized band $E_{k_{-}} \equiv E_{k}$ is occupied in the temperature range much smaller than the hybridization gap, $k_{\mathrm{B}} T \ll|V| e$. The effective Hamiltonian (1) transformed to the hybridized basis then has the form

$$
\begin{aligned}
\tilde{\mathcal{H}}_{\mathrm{SB}} & =\sum_{k \sigma} \Psi_{k_{\sigma}}^{+} E_{k^{\prime}} \Psi_{k_{\sigma}} \\
& -\frac{V^{2}}{\bar{U}} \sum_{k k^{\prime}} \frac{4 V^{2} e^{2}}{\left[\left(\varepsilon_{k}-\varepsilon_{f}\right)^{2}+4 V^{2} e^{2}\right]^{\frac{1}{2}}\left[\left(\varepsilon_{k^{\prime}}-\varepsilon_{f}\right)^{2}+4 V^{2} e^{2}\right]^{\frac{1}{2}}} \\
& \times \Psi_{k \uparrow}^{+} \Psi_{-k+q \downarrow}^{+} \Psi_{-k^{\prime} \downarrow} \Psi_{k^{\prime}+q \uparrow^{\prime}}
\end{aligned}
$$

where $\Psi_{k_{\sigma}}^{+}$is the creation operator of a hybridized $a-c$ state. Generally, $\Delta_{k} \sim V_{k}$ has nodes for $k$ points for which $V_{k}=0$. In our model situation with $V_{k}=V$ the gap is never zero; therefore, we approximate the pairing potential by its a verage over occupied quasiparticle states. This leads to an effective $\boldsymbol{k}$-independent potential

$$
\widetilde{J} \approx \frac{V^{2}}{\bar{U}}\left(\frac{k_{\mathrm{B}} T_{\mathrm{K}}}{V e}\right)^{2} \ln ^{2}\left(\frac{k_{\mathrm{B}} T_{\mathrm{K}}}{|V| e}\right)=\frac{V^{2} \rho_{0}}{4 \bar{U}} k_{\mathrm{B}} T_{\mathrm{K}} \ln ^{2}\left(k_{\mathrm{B}} T_{\mathrm{K}} \rho_{0}\right) .
$$

In the limit of $f$ electron localization $\widetilde{J} \rightarrow 0$ (note that the pairing takes place only when $e \neq 0$, i.e. when the $f$ holes exist and propagate). The disappearance of the pairing in the strict Kondo lattice limit $\left(e=0, n_{f}=1\right)$ implies that our approach indeed describes pairing, not the singlet Kondo type of state.

The local nature of pairing in conjunction with the single-band nature of the problem allows us to derive explicitly the Ginzburg-Landau functional within the Lagrangian formalism for the Grassmann variables $\Psi_{\sigma}^{+}(r)$ and $\Psi_{\sigma}(r)$ :

$$
\mathcal{L}(\tau)=\int \mathrm{d}^{3} x\left\{\sum_{\sigma} \Psi_{\sigma}^{+}\left[p_{0}+E(p)\right] \Psi_{\sigma}-\tilde{J} \Psi_{\uparrow}^{+}(x), \Psi_{\downarrow}^{+}(x) \Psi_{\downarrow}(x) \Psi_{\uparrow}(x)\right\},
$$


where $\Psi_{\sigma}=\Psi_{\sigma}(\boldsymbol{x}, \tau) p_{0} \equiv \partial_{\tau}$, and $E(\boldsymbol{p})$ is the eigenenergy $E_{k}$ with $k$ replaced by $(-\mathrm{i} \nabla)$. We also introduce the two-component Nambu notation $\Psi^{+} \equiv\left(\Psi_{\uparrow}^{+}, \Psi_{\downarrow}\right)$ in (10) and apply the Hubbard-Stratonovich transformation to the quartic term. Such procedure reduces the partition function to the form

$$
\begin{aligned}
& Z=\int \mathrm{D} \Psi \mathrm{D} \Psi^{+} \mathrm{D} \Delta \\
& \times \exp \left\{-\int_{0}^{\beta} \mathrm{d} \tau \int \mathrm{d}^{3} x\left[\Psi^{+}\left(\begin{array}{cc}
p_{0}+E(p), & -\tilde{\Delta} \\
-\tilde{\Delta}^{+}, & p_{0}-E(p)
\end{array}\right) \Psi-\frac{|\tilde{\Delta}|^{2}}{\widetilde{J}}\right]\right\},
\end{aligned}
$$

with $\tilde{\Delta}=\tilde{J} \Delta$. Integrating over the Grassmann variables and neglecting the part which does not depend explicitly on $\tilde{\Delta}$ we obtain

$$
\begin{aligned}
& Z=\int \mathrm{D} \Delta \\
& \times \exp \left\{\operatorname{Tr} \ln \left[1-\left(\begin{array}{cc}
0, & \frac{1}{p_{0}+E(\boldsymbol{p})} \tilde{\Delta} \\
\frac{1}{p_{0}-E(\boldsymbol{p})} \tilde{\Delta^{+}}, & 0
\end{array}\right)\right]-\beta \int \mathrm{d}^{3} x|\tilde{\Delta}|^{2} \tilde{J}\right\},
\end{aligned}
$$

where the part $\{\ldots\}$ is called the effective action $S_{\text {eff }}$. Expanding $\exp \left(-S_{\text {eff }}\right)$ into the Taylor series, carrying out a Fourier expansion, and evaluating corresponding sums [6] one arrives at the Ginzburg-Landau functional $F_{\mathrm{GL}}$ in the form

$$
\begin{aligned}
F_{\mathrm{GL}} & =-\frac{1}{2} \rho_{0}\left(m^{*} / m_{0}\right) \\
& \times \int_{0}^{\beta} \mathrm{d} \tau \int \mathrm{d}^{3} x\left\{\left[\left(1-\frac{T}{T_{\mathrm{c}}}\right)+\xi^{2} \nabla^{2}\right]|\tilde{\Delta}|^{2}-\frac{7}{16} \frac{\zeta(3)}{\pi^{2} T_{\mathrm{c}}^{2}}|\tilde{\Delta}|^{4}\right\},
\end{aligned}
$$

with $T_{\mathrm{c}}=1.13 D \exp (-1 / \widetilde{J} \rho(\mu)) \sim T_{\mathrm{K}}^{\frac{1}{2}} \exp \left(-k_{\mathrm{B}} T_{\mathrm{K}} / \widetilde{J}\right)$. The coherence length at $T=0$ is

$$
\xi_{0}^{2}=\frac{7}{48} \frac{\zeta(3)}{\pi^{2} T_{\mathrm{c}}^{2}}\left(\frac{k_{\mathrm{B}} T_{\mathrm{K}}}{V e}\right)^{4} \frac{k_{\mathrm{F}}^{2}}{m_{0}^{2}} \sim\left(T_{\mathrm{K}} / T_{\mathrm{c}}\right)^{2},
$$

with $k_{\mathrm{F}}$ being the Fermi wave vector and $\zeta(x)$ is the Riemann zeta function.

To determine the London penetration depth we start with the substitution $\nabla \rightarrow \nabla-2 \mathrm{i} e_{0} \boldsymbol{A} / c$, where $\boldsymbol{A}$ is the vector potential. This produces the term $(1 / 2) m_{A}^{2} A^{2}$ in $S_{\text {eff }}$, where

$$
m_{A}^{2}=\frac{2}{3} e_{0}^{2}\left(\frac{v_{\mathrm{F}}}{c^{2}}\right)^{2}\left(1-\frac{T}{T_{\mathrm{c}}}\right)\left(\frac{k_{\mathrm{B}} T_{\mathrm{K}}}{V e}\right)^{4} \rho(\mu) \sim k_{\mathrm{B}} T_{\mathrm{K}}\left(1-T / T_{\mathrm{c}}\right) m_{\mathrm{BCS}}^{2}
$$

is the photon mass in the superconducting phase, and $m_{\mathrm{BCS}}^{2}=2 e_{0}^{2}\left(v_{\mathrm{F}} / c\right)^{2} \rho_{0} / 3$ is the mass if there were no enhancement due to the presence of the $f$ level. The London penetration depth at $T=0$ is $\lambda_{0}=\left(\hbar / m_{A}\right) \sim T_{\mathrm{K}}^{-\frac{1}{2}}$. The last quantity enters the ratio $\kappa=\lambda / \xi$ which takes the form

$$
\kappa=\left\{\sqrt{2 / 3} \xi_{0} e_{0} v_{\mathrm{F}}\left(\tilde{\varepsilon}_{f} /|V| e\right) \sqrt{\rho_{0}}\right\}^{-1} \sim T_{\mathrm{K}}^{-3 / 2} T_{\mathrm{c}} .
$$

Note that we have used the relation $\xi \equiv \xi(T)=\xi_{0} /\left(1-T / T_{\mathrm{c}}\right)^{\frac{1}{2}}$. Close to $f$ electron localization $T_{\mathrm{K}} \rightarrow 0$ and then $\kappa \gg 1$. 
The expression (13) can be used to determine the thermodynamic critical magnetic field $B_{\mathrm{c}}$ via the relation $B_{\mathrm{c}}^{2} / 2=-F_{\mathrm{GL}} / V_{0}$, where $V_{0}$ is the volume of the system. Explicitly,

$$
B_{\mathrm{c}}=\left[\frac{2 \pi^{2}}{7 \zeta(3)} \rho(\mu)\right]^{\frac{1}{2}}\left(T_{\mathrm{c}}-T\right) \sim\left(T_{\mathrm{c}}-T\right) T_{\mathrm{K}}^{-\frac{1}{2}}
$$

and therefore the first and second critical magnetic fields are

$$
B_{\mathrm{cl}}=\frac{\Phi_{0}}{2 \pi \lambda^{2}} \ln (\lambda / \xi) \sim T_{\mathrm{K}} \ln \left(T_{\mathrm{K}}^{-3 / 2} T_{\mathrm{c}} a\right),
$$

where $a$ is a constant, and

$$
B_{\mathrm{c} 2}=\sqrt{2} \kappa B_{\mathrm{c}}=2\left[\frac{3 \pi^{2}}{7 \zeta(3)}\right]^{\frac{1}{2}} \frac{1}{\xi_{0} e v_{\mathrm{F}}}\left(\frac{V e}{\tilde{\varepsilon}_{f}}\right)^{2} \sim\left(T_{\mathrm{c}} / T_{\mathrm{K}}^{2}\right)\left(T_{\mathrm{c}}-T\right) .
$$

We have calculated the derivative $B_{\mathrm{c} 2}^{\prime}=-\left(\mathrm{d} B_{\mathrm{c} 2} / \mathrm{d} T\right)$ at $T=T_{\mathrm{c}}$ and have plotted it as a function of $T_{\mathrm{c}} \gamma^{2}$, as well as $B_{\mathrm{c} 1}^{\prime}$ at $T_{\mathrm{c}}$ as displayed for various systems in Fig. $1 \mathrm{a}$ and $\mathrm{b}$. This figures indicate that heavy-fermion superconductors come out
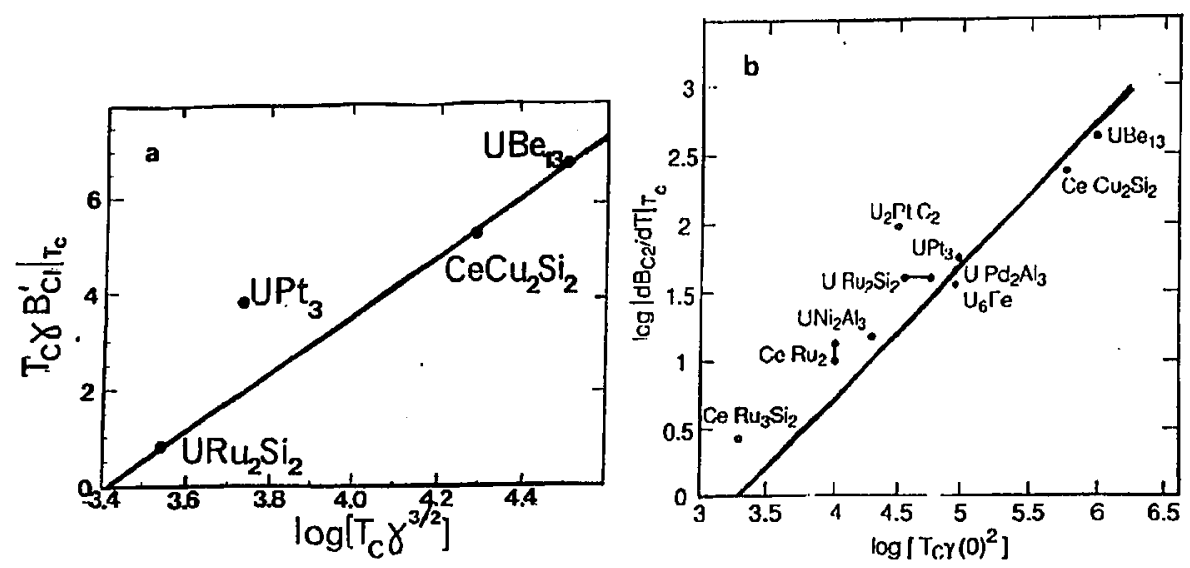

Fig. 1. Predicted linear scaling of first (a) and second (b) critical field derivatives at $T=T_{\mathrm{c}}$ for various heavy-fermion superconductors (solid line). The solid line represents the result coming from the Ginzburg-Landau-Gorkov theory in the clean limit. For explanation see main text.

in clean limit rather than dirty. The data were extracted from the works listed in Ref. [7]. However, it is important to note that the proposed scaling is independent of pairing mechanism, as the coupling constant appears only via $T_{\mathrm{c}}$.

In summary, we have presented a universal scaling picture for superconducting phase of heavy fermions, taking into account processes of the order $V^{2} / U$ which produce the pairing. Even though $F_{\mathrm{GL}}$ is of standard form, the coefficients acquire unusually high values because of the factor $m^{*} / m_{0}$ Eq. (10).

The authors acknowledge the support of the Committee for Scientific Research, grant No. 204299101. 


\section{References}

[1] A.J. Millis, P.A. Lee, Phys. Rev. B 35, 3394 (1987); A. Auerbach, K. Levin, Phys. Rev. B 34, 354 (1986).

[2] J. Spałek, Phys. Rev. B 38, 208 (1988); J. Spalek, J.M. Honig, in: Studies of High-Temperatures Superconductors, Vol. 8, Ed. A. Narlikar, Nova Sci., New York 1991, p. 1.

[3] M. Lavagna, A. Millis, P.A. Lee, Phys. Rev. Lett. 58, 255 (1987).

[4] Z. Zou, P.W. Anderson, Phys: Rev. B 37, 627 (1988).

[5] A model with $\left\langle d_{i}^{+}\right\rangle \neq 0$ has been considered by D.M. Newns, Phys. Rev. B 36, 2429 (1987).

[6] H. Kleinert, Fortschr. Phys. 26, 565 (1978); A.M.J. Schakel, Ph.D. Thesis, University of Amsterdam, 1989, unpublished.

[7] For $B_{\mathrm{c} 2}^{\prime}$ : UBe $\mathrm{UB}_{13}$ : M.B. Maple, J.W. Chen, S.E. Lambert, Z. Fisk, J.L. Smith, H.R. Ott, J.S. Brooks, M.J. Naughton, Phys. Rev. Lett. 54, 477 (1985); $\mathrm{CeCu}_{2} \mathrm{Si}_{2}$ : W. Assmus, M. Herrmann, U. Rauchschwalbe, S. Riegel, W. Lieke, H. Spille, S. Horn, G. Weber, F. Steglich, G. Cordier, ibid. 52, 469 (1984); $\mathrm{UPt}_{3}$ averaged: J.W. Chen, S.E. Lambert, M.B. Maple, Z. Fisk, J.L. Smith, G.R. Stewart, J.O. Willis, Phys. Rev. B 30, 1583 (1984); UPd $\mathrm{Al}_{3}$ : C. Geibel, C. Schank, S. Thies, H. Kitazawa, C.D. Bredl, A. Böhm, M. Rau, A. Grauel, R. Caspary, R. Helfrich, U. Ahlheim, G. Weber, F. Steglich, Z. Phys. B 84, 1 (1991); U6 Fe: L.E. Delong, G.W. Crabtree, L.N. Hall, H. Kierstead, H. Aoki, S.K. Dhar, K.A. Gschneidner Jr., A. Junod, Physica B $+C$ 135, 81 (1985); URu $\mathrm{Si}_{2}$ : W. Schlabitz, J. Baumann, B. Pollit, U. Rauchschwalbe, H.M. Mayer, U. Ahlheim, C.D. Bredl, Z. Phys. B 62, 171 (1986) and M.B. Maple, Y. Dalichaouch, B.W. Lee, C.L. Seaman, P.K. Tsai, P.E. Armstrong, Z. Fisk, C. Rossel, M.S. Torikachvili, Physica B 171, 219 (1991); $\mathrm{U}_{2} \mathrm{PtC}_{2}$ : G.P. Meisner, A.L. Giorgi, A.C. Lawson. G.R. Stewart, J.O. Willis, M.S. Wire, J.L. Smith, Phys. Rev. Lett. 53, 1829 (1984); $\mathrm{UNi}_{2} \mathrm{Al}_{3}$ : C. Geibel, S. Thies, D. Kaczorowski, A. Mehner, A. Grauel, B. Seidel, U. Ahlheim, R. Helfrich, K. Petersen, C.D. Bredl, F. Steglich, Z. Phys. B 83, 305 (1991); $\mathrm{CeRu}_{3} \mathrm{Si}_{2}$ : U. Rauchschwalbe, W. Lieke, F. Steglich, C. Godart, L.C. Gupta, R.D. Parks, Phys. Rev. B 30, 444 (1984). For $B_{\mathrm{c} 1}^{\prime}$ : $\mathrm{UBe}_{13}$ : E.A. Knetsch, J.A. Mydosh, R.H. Heffner, J.L. Smith, Physica B 163, 209 (1990); $\mathrm{CeCu}_{2} \mathrm{Si}_{2}$ : U. Rauchschwalbe, Physica $B+C$ 147, 1 (1987); UPt U $_{3}$ B.S. Shivaram, J.J. Gannon Jr., D.G. Hinks, Physica $B$ 163, 629 (1990) and S. Wüchner, N. Keller, J.L. Tholence, J. Flouquet, Solid State Commun. 85, 355 (1993); URu $\mathrm{Si}_{2}$ : U. Rauchschwalbe, Physica $B+C$ 147, 1 (1987) and S. Wüchner, N. Keller, J.L. Tholence, J. Flouquet, Solid State Commun. 85, 355 (1993). 